\title{
A New Compound Fréchet Distribution for Modeling Breaking Stress and Strengths Data
}

\author{
Haitham M. Yousof \\ Department of Statistics, Mathematics and Insurance, Benha University, Egypt. \\ Email: haitham.yousof@fcom.bu.edu.eg \\ Nadeem Shafique Butt \\ Department of Family and Community Medicine King Abdul Aziz University, Jeddah, \\ Kingdom of Saudi Arabia. \\ Email: nshafique@kau.edu.sa \\ Refah Mohammed Alotaibi \\ Mathematical Sciences Department, College of Science, Princess Nourah bint Abdulrahman \\ University, Riyadh, Saudi Arabia. \\ Email: rmalotaibi@pnu.edu.sa \\ Rezk H. \\ Mathematical Sciences Department, College of Science, Princess Nourah bint Abdulrahman \\ University, Riyadh, Saudi Arabia and Department of Statistics, Al-Azhar University, Cairo, \\ Egypt. \\ Email: hrmohamed@pnu.edu.sa \\ Ghadah A. Alomani \\ Mathematical Sciences Department, College of Science, Princess Nourah bint Abdulrahman \\ University, Riyadh, Saudi Arabia. \\ Email: gaalomani@pnu.edu.sa \\ Mohamed Ibrahim \\ Department of Applied Statistics and Insurance, Faculty of Commerce, Damietta University, \\ Egypt. \\ Email: mohamed_ibrahim@du.edu.eg
}

\begin{abstract}
A new useful version of the Fréchet model is introduced and studied. Some of its properties are derived. The method of maximum likelihood is used for estimating the unknown parameter via two real data applications. The new version is much better than other important competitive Fréchet models in modeling two real data sets.
\end{abstract}

Keywords: Zero-Truncated Poisson Distribution; Fréchet Distribution; Generating Function, Maximum Likelihood; Breaking Stress; Strengths Data.

\section{Introduction and physical motivation}

A random variable (RV) $W$ has the one parameter Fréchet $(\mathrm{Fr})$ model if its probability density function (PDF) $\left(\mathbf{g}_{\beta}(w)\right)$, survival function $(\mathrm{SF})\left(\overline{\mathbf{G}}_{\beta}(w)\right)$, cumulative distribution function $(\mathrm{CDF})\left(\mathbf{G}_{\beta}(w)\right)$ and hazard rate function (HR_F) $\left(\boldsymbol{\tau}_{\beta}(w)\right)$ are given by (for $w \geq$ 0 )

$$
\mathbf{g}_{\beta}(w)=\beta w^{-\beta-1} \exp \left(-w^{-\beta}\right)
$$




$$
\mathbf{G}_{\beta}(w)=\exp \left(-w^{-\beta}\right), \overline{\mathbf{G}}_{\beta}(w)=1-\mathbf{G}_{\beta}(w)
$$

and

$$
\boldsymbol{\tau}_{\beta}(w)=1-\exp \left(-w^{-\beta}\right)
$$

respectively. The parameter $\beta>0$ control the shape of the model. In this paper we study a new Fr version using compounding technique with the discrete zero-truncated-Poisson (ZTcP) model. The probability mass function (PM_F) of the ZTcP model is given by

$$
\left.\mathbf{P}(\mathbf{M}=m)\right|_{(m=1,2, \ldots)}=\frac{\lambda^{m} \overline{\mathbf{e x p}}(-\lambda)}{m ! \mathbf{Y}_{(\lambda)}} \text {. }
$$

where $\mathbf{Y}_{(\lambda)}=-\exp (-\lambda)+1$. If $\mathbf{M}$ has the ZTcP model, then

$$
\mathbf{E}(\mathbf{M} \mid \lambda)=\lambda / \mathbf{Y}_{(\lambda)}
$$

and

$$
\mathbf{V} \mathbf{a} \mathbf{r}(\mathbf{M} \mid \lambda)=\frac{\lambda}{\mathbf{Y}_{(\lambda)}}\left[1+\lambda-\frac{\lambda}{\mathbf{Y}_{(\lambda)}}\right]
$$

where $\mathbf{E}(\mathbf{M} \mid \lambda)$ is the expected-value and $\operatorname{Var}(\mathbf{M} \mid \lambda)$ is Variance of the RV W. Consider the model Burr X Fr $(\operatorname{BXFr}(\theta, \beta))$ defined by the $\operatorname{CDF}\left(H_{\theta, \beta}(z)\right)$ and $\operatorname{PDF}\left(h_{\theta, \beta}(w)\right)$ given by

$$
\mathbf{H}_{\theta, \beta}(w)=\left(1-\exp \left\{-\left[\exp \left(w^{-\beta}\right)-1\right]^{-2}\right\}\right)^{\theta}
$$

and

$$
\begin{aligned}
& \mathbf{h}_{\theta, \beta}(w)=2 \theta \beta\left[1-\exp \left(-w^{-\beta}\right)\right]^{-3} \\
& \times \exp \left[-2\left(w^{-\beta}\right)\right] \exp \left\{-\left[\exp \left(w^{-\beta}\right)-1\right]^{-2}\right\} \\
& \times w^{-(1+\beta)}\left(1-\exp \left\{-\left[\exp \left(w^{-\beta}\right)-1\right]^{-2}\right\}\right)^{\theta-1} .
\end{aligned}
$$

respectively, where $\alpha>0, \theta>0$ and $\beta>0$. Let $F_{j}$ be the failure (death) time of any subsystem from a certain system, where

$$
\min \left\{F_{j} \mid j=1,2, \ldots, \mathrm{M}\right\}=W
$$

Then, the conditional CDF for the RV $W \mid M$ will be

$$
F(w \mid M)=1-\operatorname{Pr}(W>w \mid M)=1-\left[1-\mathbf{H}_{\theta, \beta}(w)\right]^{M} .
$$


Then, the PBX-Fr density function, can be formulated as

$$
\left.F_{\lambda, \theta, \beta}(w)\right|_{(\lambda \in R-\{0\})}=\frac{1-\exp \left[-\lambda\left(1-\exp \left\{-\left[\exp \left(w^{-\beta}\right)-1\right]^{-2}\right\}\right)^{\theta}\right]}{\mathbf{Y}_{(\lambda)}},
$$

with the corresponding PDF

$$
\begin{aligned}
& \left.f_{\lambda, \theta, \beta}(w)\right|_{(\lambda \in R-\{0\})}=2 \theta \lambda \beta \mathbf{Y}_{(\lambda)} w^{-(\beta+1)}\left\{1-\exp \left(-w^{-\beta}\right)\right\}^{-3} \\
& \times \exp \left(-2 w^{-\beta}-\left[\exp \left(w^{-\beta}\right)-1\right]^{-2}\right) \\
& \times\left[1-\exp \left(-\left[\exp \left(w^{-\beta}\right)-1\right]^{-2}\right)\right]^{\theta-1} \\
& \times \exp \left\{-\lambda\left[1-\exp \left(-\left[\exp \left(w^{-\beta}\right)-1\right]^{-2}\right)\right]^{\theta}\right\}
\end{aligned}
$$

The HR_F of the new model can be calculated via

$$
\tau_{\lambda, \theta, \beta}(w)=f_{\lambda, \theta, \beta}(w) /\left[1-F_{\lambda, \theta, \beta}(w)\right] .
$$

The PDF of the PBX-Fr model can be right skewed and unimodal with different shapes (see Figure 1) also it can be left skewed (see Table 1). The HR_F of the PBX-Fr model can be bathtub (the $\mathbf{U}$-shape), upside-down-bathtub (upside-down-U), increasing-constantincreasing, decreasing and constant. 

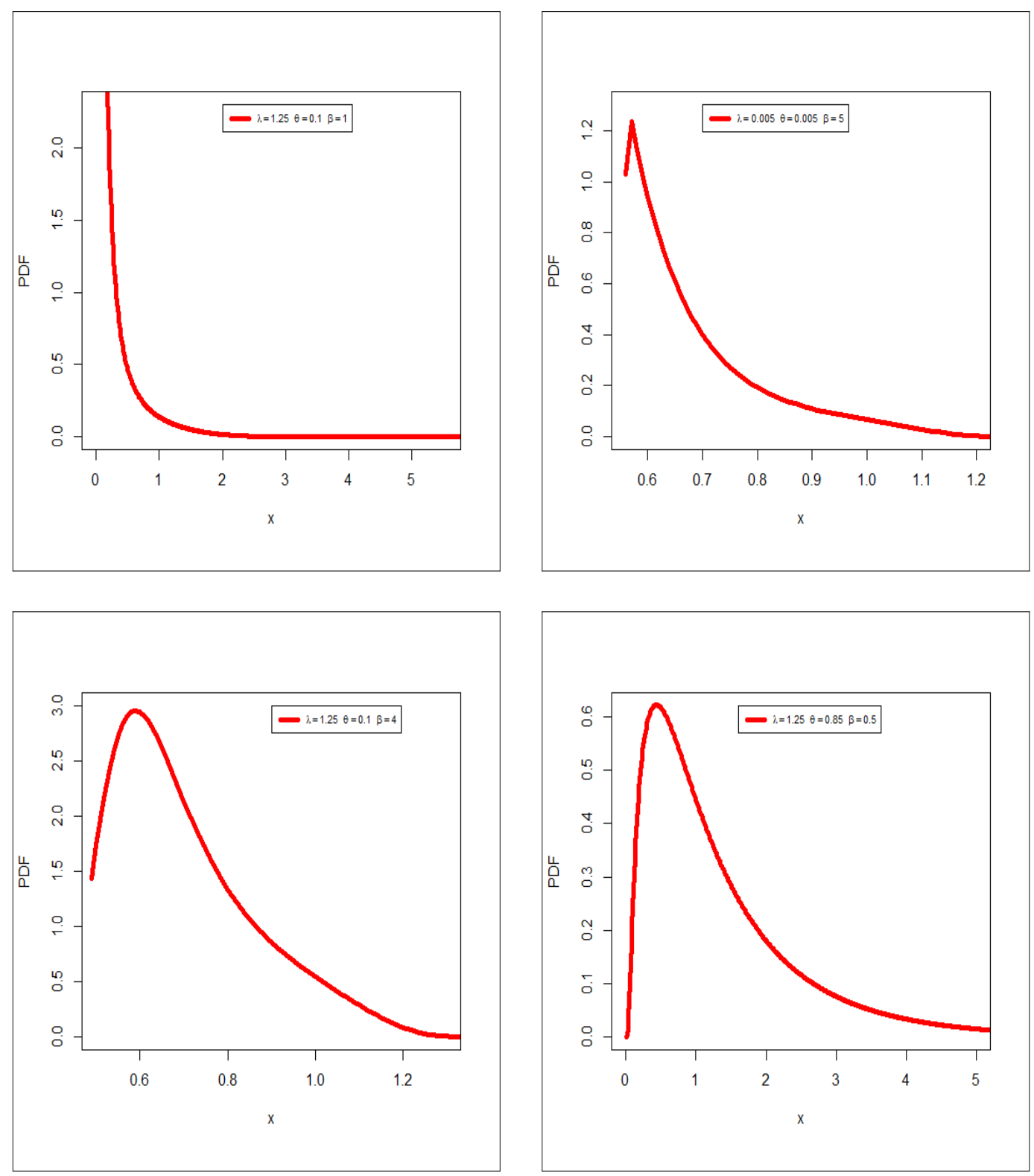

Figure 1:PDFs 

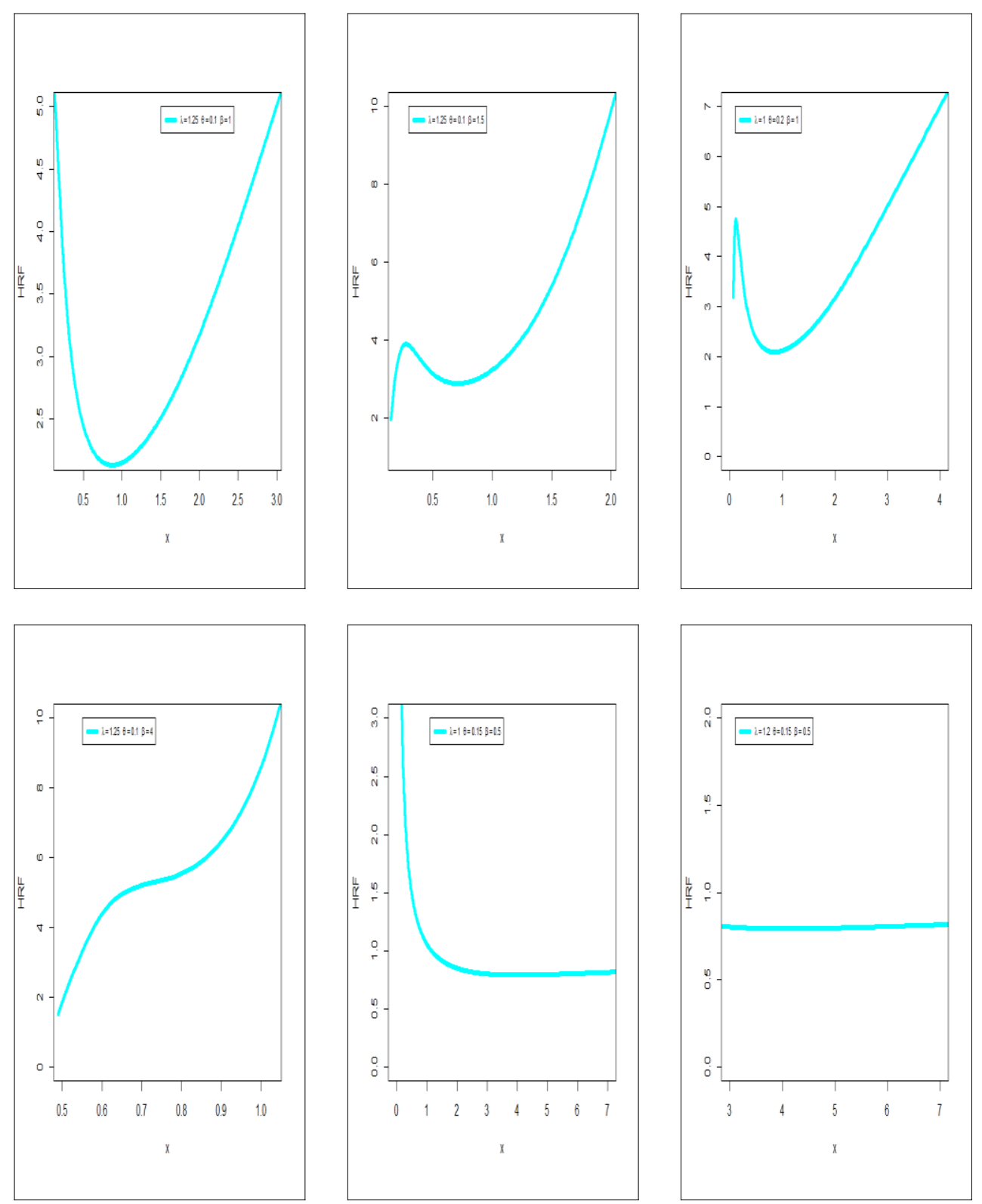

Figure 2: HRFs

Some important extensions of the Fr model have been developed and studied such as Nadarajah. and Kotz (2003), Mead.etal., (2014), Yousof.etal., (2015), Afify.etal., (2016_a), Afify.et al., (2016_b), Yousof.et al., (2016), Korkmaz.et al., (2017), Brito. et al., (2017), Hamedani.etal., (2017), Cordeiro.etal., (2018), Yousof.etal., (2018_ a), Chakraborty.etal., (2018), Hamedani. etal., (2018), Korkmaz.etal., (2018), Yousof.etal., (2018_b), Hamedani.et al., (2019) and Korkmaz.etal., (2019), among others. 


\section{Properties}

\subsection{Some expansions}

Using

$$
\exp (\boldsymbol{v})=\sum_{q=0}^{\infty} \boldsymbol{v}^{q} \frac{1}{q !}
$$

the PDF in (6) can be written as

$$
\begin{aligned}
& f_{\lambda, \theta, \beta}(w)=\sum_{\omega=0}^{\infty} 2(-1)^{\omega} \theta \beta \frac{\lambda^{1+\omega}}{\omega ! \mathbf{Y}_{(\lambda)}} \\
& \times \exp \left\{-\left[\exp \left(w^{-\beta}\right)-1\right]^{-2}\right\} \\
& \times \frac{w^{-(\beta+1)} \exp \left[-2 \exp \left(-w^{-\beta}\right)\right]}{\left[-\exp \left(-w^{-\beta}\right)+1\right]^{3}} \\
& \times\left(1-\exp \left\{-\left[\exp \left(w^{-\beta}\right)-1\right]^{-2}\right\}\right)^{-1+(\omega+1) \theta} .
\end{aligned}
$$

Consider the following power series

$$
\left(1-\frac{s}{b}\right)^{\mu}=\sum_{\omega=0}^{\infty} \frac{\left(-\frac{s}{b}\right)^{\omega} \Gamma(1+\mu)}{\Gamma(1+\omega) \Gamma(1+\mu-\omega)^{\prime}}
$$

where $\left|\frac{s}{b}\right|<1$ and $\mu>0$ is a real but non-integer. Applying (8) to

$$
\left\{1-\exp \left[-\left[\exp \left(w^{-\beta}\right)-1\right]^{-2}\right]\right\}^{(\omega+1) \theta-1}
$$

in (7) we have

$$
\begin{aligned}
& f_{\lambda, \theta, \beta}(w)=\theta \beta \frac{2}{\mathbf{\Upsilon}_{(\lambda)}} x^{-(\beta+1)} \exp \left[-2 w^{-\beta}\right] \\
& \times \sum_{\omega, \tau=0}^{\infty}(-1)^{\omega+\tau} \frac{\lambda^{1+\omega} \Gamma((\omega+1) \theta)}{\tau ! \Gamma((\omega+1) \theta-\tau)} \\
& \times \frac{\exp \left\{-(\tau+1)\left[\exp \left(w^{-\beta}\right)-1\right]^{-2}\right\}}{\left[1-\exp \left(-w^{-\beta}\right)\right]^{3}} .
\end{aligned}
$$

Applying the $\exp (\boldsymbol{v})$ series to

$$
\exp \left[-(\tau+1)\left[\exp \left(w^{-\beta}\right)-1\right]^{-2}\right]
$$


expression (9) becomes

$$
\begin{aligned}
& f_{\lambda, \theta, \beta}(w)=2 \beta w^{-(\beta+1)} \exp \left(-w^{-\beta}\right) \\
& \times \sum_{\omega, \tau, v=0}^{\infty}(-1)^{\omega+\tau+v} \frac{\theta \lambda^{1+\omega}(\tau+1)^{v} \Gamma((\omega+1) \theta)}{\tau ! v ! \mathbf{Y}_{(\lambda)} \Gamma((\omega+1) \theta-\tau)} \\
& \times \frac{\left[\exp \left(-w^{-\beta}\right)\right]^{2 v+1}}{\left[1-\exp \left(-w^{-\beta}\right)\right]^{2 v+3}} .
\end{aligned}
$$

Consider

$$
\left.(1-\boldsymbol{p})^{-\mu}\right|_{(|\boldsymbol{p}|<1 \text { and } \mu>0)}=\sum_{\kappa=0}^{\infty} \boldsymbol{p}^{\kappa} \frac{\Gamma(\mu+\kappa)}{\Gamma(1+\kappa) \Gamma(\mu)}
$$

Applying (11) to (10) then (10) becomes

$$
\begin{gathered}
f_{\lambda, \theta, \beta}(w)=\sum_{\omega, \tau, v, \kappa=0}^{\infty} 2 \theta \lambda^{1+\omega}(-1)^{\omega+\tau+v} \\
\times \frac{(\tau+1)^{v}}{\Gamma(1+\kappa) \mathbf{Y}_{(\lambda)}[2(1+v)+\kappa]} \\
\times \frac{\Gamma((\omega+1) \theta) \Gamma(3+2 v+\kappa)}{\tau ! v ! \Gamma((\omega+1) \theta-\tau) \Gamma(2 v+3)}[2(1+v)+\kappa] \\
\times \beta x^{-(\beta+1)} \exp \left\{-[2(1+v)+\kappa]\left(x^{-1}\right)^{\beta}\right\},
\end{gathered}
$$

which can be written as

$$
f_{\lambda, \theta, \beta}(w)=\sum_{\nu, \kappa=0}^{\infty} v_{v, \kappa} \pi_{[2(1+v)+\kappa]}(w ; \beta),
$$

where

$$
\begin{gathered}
v_{v, \kappa}=2 \theta \lambda^{1+\omega}(-1)^{v} \frac{\Gamma(3+2 v+\kappa)}{v ! \Gamma(1+\kappa) \mathbf{Y}_{(\lambda)} \Gamma(2 v+3)[2(1+v)+\kappa]} \\
\times \sum_{\omega, \tau=0}^{\infty}(-1)^{\omega+\tau} \frac{\Gamma((\omega+1) \theta)(\tau+1)^{v}}{\tau ! \Gamma((\omega+1) \theta-\tau)}
\end{gathered}
$$

and the density $\pi_{[2(1+v)+\kappa]}(w ; \beta)$ is the Fr PDF with scale $\sqrt[\beta]{\kappa+2(1+v)}$ and $\beta$ as a shape parameter. By the same technique, we have 


$$
F_{\lambda, \theta, \beta}(w)=\sum_{v, \kappa=0}^{\infty} v_{v, \kappa} \Pi_{[2(1+v)+\kappa]}(x ; \beta)
$$

where the $\operatorname{CDF} \Pi_{[2(1+v)+\kappa]}(w ; \beta)$ is the the Fr CDF with scale parameter $\sqrt[\beta]{\kappa+2(1+v)}$ and $\beta$ as a shape parameter and

$$
\frac{d}{d w} \sum_{\nu, \kappa=0}^{\infty} v_{v, \kappa} \Pi_{[2(1+v)+\kappa]}(w ; \beta)=\sum_{v, \kappa=0}^{\infty} v_{v, \kappa} \pi_{[2(1+v)+\kappa]}(w ; \beta) .
$$

\subsection{Quantile function (Q_F)}

The Q_F of $W$, where $W \sim \operatorname{PBX}-\operatorname{Fr}(\lambda, \theta, \beta)$, is obtained by inverting (5) as

$$
\mathbf{Q}(u)=\sqrt[\beta]{\left\{-\ln \left[\left(1+\sqrt{\left.-\ln \left\{-\sqrt{\frac{-\ln \left[1-u \mathbf{Y}_{(\lambda)}\right]}{\lambda}+1}\right\}\right)} .\right.\right.\right.} .
$$

\subsection{Raw moments}

The q-th raw moment of $X$, say $\mu_{r}^{\prime}$, comes from (12) as

$$
\left.\mu_{\mathbf{q}}^{\prime}\right|_{(\mathbf{q}<\beta)}=\mathbf{E}\left(W^{\mathbf{q}}\right)=\sum_{\nu, \kappa=0}^{\infty} v_{v, \kappa}[2(1+v)+\kappa]^{\frac{\mathbf{q}}{\beta}} \Gamma\left(1-\frac{\mathbf{q}}{\beta}\right) .
$$

Setting $\mathbf{q}=1$ in (14) gives the mean of $W$ as

$$
\left.\mathbf{E}(W)\right|_{(1<\beta)}=\sum_{v, \kappa=0}^{\infty} v_{v, \kappa} \sqrt[\beta]{2(1+v)+\kappa} \Gamma\left(1-\frac{1}{\beta}\right)
$$

where

$$
\Gamma(1+\mathbf{v})=\mathbf{v} !=\prod_{u=0}^{\mathbf{v}-1}(\mathbf{v}-u)
$$

and

$$
\int_{0}^{\infty} w^{\mathbf{v}-1} \exp (-w) d w=\Gamma(\mathbf{v})
$$

The "Bowley's skewness" is given by 


$$
\text { Bowley's Skewness }=\left[\mathbf{Q}_{\left(\frac{3}{4}\right)}-2 \mathbf{Q}_{\left(\frac{2}{4}\right)}+\mathbf{Q}_{\left(\frac{1}{4}\right)}\right] /\left[\mathbf{Q}_{(*)}\right]
$$

and the "Moors's kurtosis" is

$$
\text { Moors's Kurtosis }=\left[\mathbf{Q}_{\left(\frac{7}{8}\right)}-\mathbf{Q}_{\left(\frac{5}{8}\right)}+\mathbf{Q}_{\left(\frac{3}{8}\right)}-\mathbf{Q}_{\left(\frac{1}{8}\right)}\right] /\left[\mathbf{Q}_{(*)}\right]
$$

Where $\mathbf{Q}_{(*)}=\left[-\mathbf{Q}_{\left(\frac{1}{4}\right)}+\mathbf{Q}_{\left(\frac{3}{4}\right)}\right]$ Plots of the PBX-Fr skewness and kurtosis is presented in Figure 3 and 4. This plot indicates that skewness and kurtosis depend on the shape parameters $\theta$ and $\beta$ where $\lambda=2$.

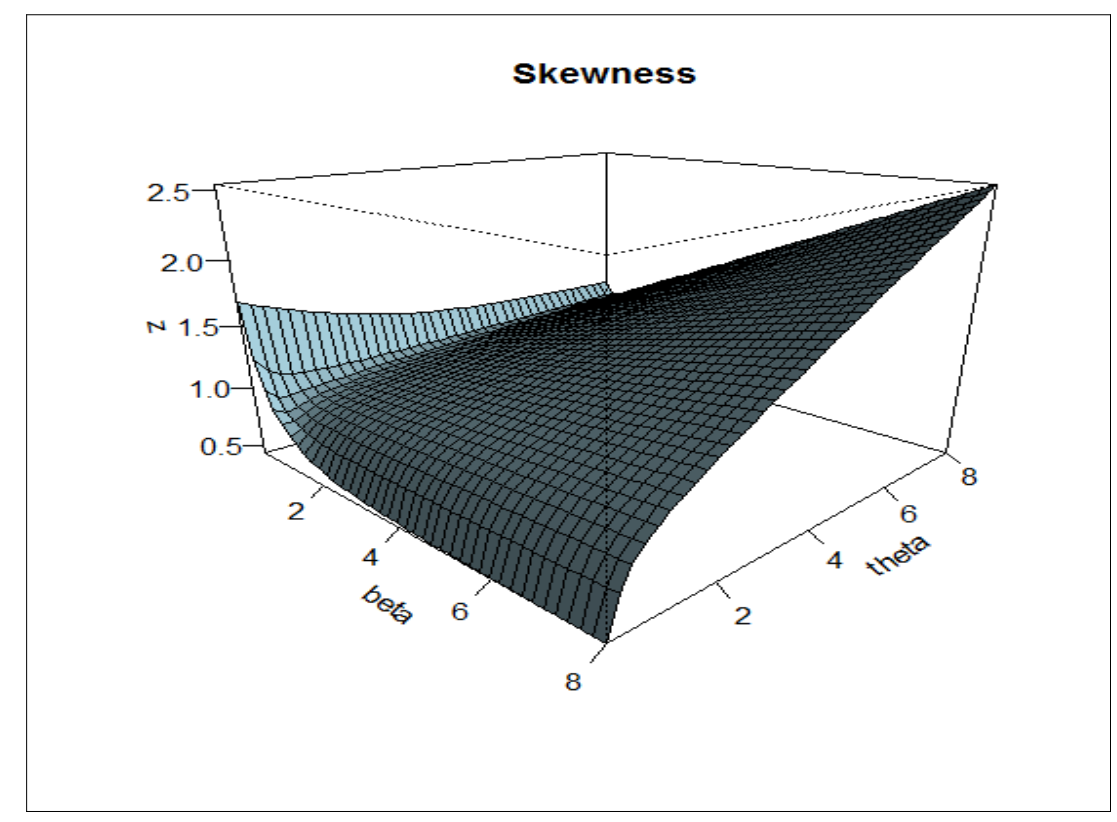

Figure 3: Plot of skewness of new distribution. 


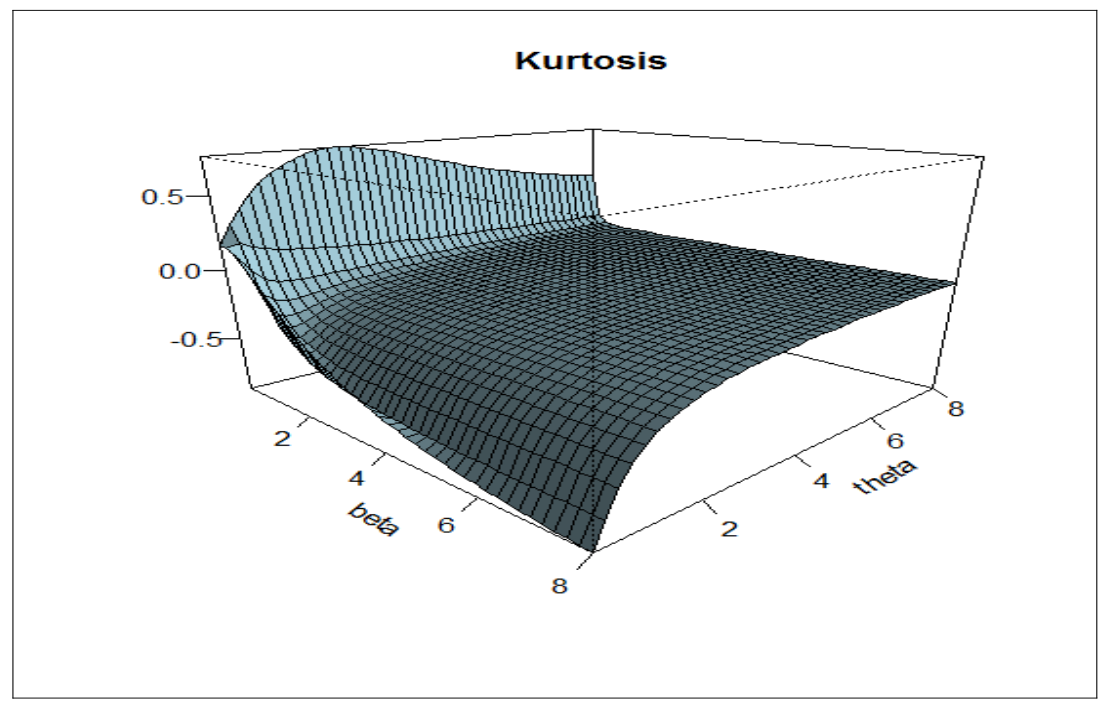

Figure 4: Plot of kurtosis of new distribution.

Numerically, the skewness of the PBX-Fr distribution can be positive and negative as well, whereas, the kurtosis of the PBX-Fr model varies in the interval $(3.1,3.9)$ also the mean of $W$ decreases as $\lambda$ and $\beta$ increases and increases as $\theta$ increases, all numerical results are listed in Table 1 below.

Table 1: Mean, variance, skewness and kurtosis of the PBX-Fr distribution with different values the parameters.

\begin{tabular}{|c|c|c|c|c|c|c|}
\hline$\lambda$ & $\theta$ & $\beta$ & Mean & Variance & Skewness & Kurtosis \\
\hline-500 & 10 & 5 & 1.283237 & 0.00022743 & 0.6961859 & 3.813617 \\
\hline-100 & & & 1.261362 & 0.00031634 & 0.6098402 & 3.628137 \\
\hline-50 & & & 1.250562 & 0.00037508 & 0.5538569 & 3.531990 \\
\hline-5 & & & 1.203300 & 0.00094420 & -0.0677616 & 3.556737 \\
\hline-1 & & & 1.172023 & 0.00143736 & -0.0062413 & 3.050183 \\
\hline 1 & & & 1.150805 & 0.00138586 & 0.2608065 & 3.173515 \\
\hline 5 & & & 1.120733 & 0.00079770 & 0.2944354 & 3.866787 \\
\hline 50 & & & 1.078752 & 0.00027670 & -0.5549989 & 3.594443 \\
\hline 100 & & & 1.069484 & 0.00023312 & -0.6328713 & 3.718253 \\
\hline 500 & & & 1.050705 & 0.00017125 & -0.7484893 & 3.991212 \\
\hline 10 & 1 & 2.5 & 0.838664 & 0.01038740 & 0.0840553 & 3.100978 \\
\hline & 5 & & 1.135617 & 0.00387498 & -0.1273857 & 3.398661 \\
\hline & 10 & & 1.222138 & 0.00246330 & -0.0687633 & 3.347035 \\
\hline & 50 & & 1.366129 & 0.00105489 & 0.07168144 & 3.534765 \\
\hline & 100 & & 1.412847 & 0.00079321 & 0.1214303 & 3.585279 \\
\hline & 500 & & 1.500865 & 0.00046736 & 0.2065899 & 3.70187 \\
\hline & 1000 & & 1.532607 & 0.00038836 & 0.2336289 & 3.708488 \\
\hline 100 & 100 & 0.1 & 2728.376 & 640425.500 & 0.3863735 & 3.160812 \\
\hline & & 0.5 & 4.831184 & 0.08679921 & -0.328818 & 3.176079 \\
\hline & & 1 & 2.196960 & 0.00455308 & -0.4271095 & 3.321732 \\
\hline & & 2 & 1.482038 & 0.00052200 & -0.4774806 & 3.409141 \\
\hline & & 3 & 1.299855 & 0.00017894 & -0.4944991 & 3.442545 \\
\hline
\end{tabular}




\subsection{Incomplete moments}

The $\mathbf{q}$-th incomplete moment $\left(v_{\mathbf{q}}(\mathbf{y})\right)$ of $W$ is

$$
v_{\mathbf{q}}(\mathbf{y})=\int_{-\infty}^{\mathbf{y}} w^{\mathbf{q}} f(w) d w
$$

We can write from (12)

$$
\left.v_{\mathbf{q}}(\mathbf{y})\right|_{(\mathbf{q}<\beta)}=\sum_{\nu, \kappa=0}^{\infty} v_{v, \kappa}[2(1+v)+\kappa]^{\frac{\mathbf{q}}{\beta}} \gamma\left(1-\frac{\mathbf{q}}{\beta},\left(\frac{1}{\mathbf{y}}\right)^{\beta}\right) .
$$

Setting $\mathbf{q}=1$ in (15) gives the 1 st $\left(v_{\mathbf{q}=\mathbf{1}}(\mathbf{y})\right)$ incomplete moment of $X$ as

$$
\left.v_{1}(\mathbf{y})\right|_{(1<\beta)}=\sum_{\nu, \kappa=0}^{\infty} v_{v, \kappa} \sqrt[\beta]{2(1+v)+\kappa} \gamma\left(1-\frac{1}{\beta},\left(\frac{1}{\mathbf{y}}\right)^{\beta}\right)
$$

where $\gamma(v, q)$ is the incomplete gamma function

$$
\begin{aligned}
& \left.\gamma(\mathbf{v}, q)\right|_{(\mathbf{v} \neq 0,-1,-2, \ldots)}=\int_{0}^{q} w^{\mathbf{v}-1} \exp (-w) d w \\
& =\frac{1}{v_{\infty}}\left\{1 \mathbf{F}_{1}[\mathbf{v} ; \mathbf{v} a+1 ;-q]\right\} q^{\mathbf{v}} \\
& =\sum_{\kappa=0}^{\infty} q^{\mathbf{v}+\kappa} \frac{(-1)^{\kappa}}{\kappa !(\mathbf{v}+\kappa)}
\end{aligned}
$$

the function $1 \mathbf{F}_{1}[\cdot ; \cdot ; \cdot]$ is a called the confluent hypergeometric function,

$$
\left.\Gamma(\mathbf{v}, q)\right|_{(w>0)}=\int_{q}^{\infty} w^{\mathbf{v}-1} \exp (-w) d w
$$

and

$$
\Gamma(\mathbf{v}, q)+\gamma(\mathbf{v}, q)=\Gamma(\mathbf{v})
$$

\subsection{Generating function (GF)}

The GF, say $\mathbf{G F}(t)=\mathbf{E}(\exp (t W))$, is obtained from (12) as 


$$
\left.\mathbf{G F}(t)\right|_{(r<\beta)}=\sum_{\nu, \kappa, r=0}^{\infty} v_{v, \kappa}\left(t^{r} / r !\right)[2(1+v)+\kappa]^{\frac{r}{\beta}} \Gamma\left(1-\frac{r}{\beta}\right)
$$

Consider the Wright hypergeometric function (the generalized case) $\left([p] \Psi_{[q]}\right)$, them

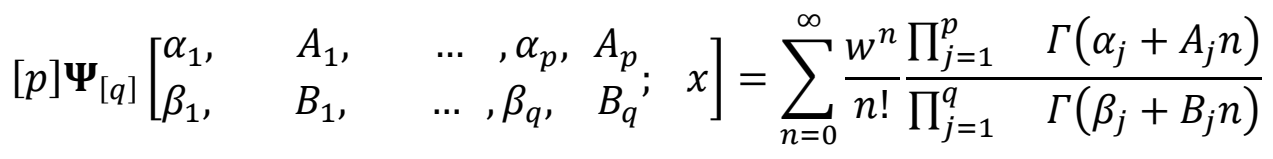

Then, we can write $\mathbf{G F}_{W}(t ; \beta)$ as

$$
\mathbf{G F}_{W}(t ; \beta)={ }_{[1]} \boldsymbol{\Psi}_{[0]}\left[\left(1,-\frac{1}{\beta}\right) ; t\right] .
$$

Combining expressions (12) and (16), we obtain the GF of $W, \mathbf{G F}_{W}(t)$, as

$$
\mathbf{G F}_{W}(t)=\sum_{\nu, \kappa=0}^{\infty} v_{v, \kappa}\left\{[1] \Psi_{[0]}\left[\left(1,-\frac{1}{\beta}\right) ; t \sqrt[\beta]{2(1+v)+\kappa}\right]\right\}
$$

\section{Estimation}

Consider a random sample (RS) from the size $K$ our PBX-Fr version. Then, the loglikelihood-function $\left(\ell_{(\lambda, \theta, \beta)}\right)$ can be expressed as

$$
\begin{aligned}
\ell_{(\lambda, \theta, \beta)} & =K \log 2+K \log \theta+K \log \lambda+K \log \beta+K \beta \log \alpha \\
-K & \log \mathbf{\Upsilon}_{(\lambda)}-(\beta+1) \sum_{h=1}^{K} \log w_{h}-3 \log \left(1-s_{i}\right) \\
& +2 \sum_{h=1}^{K} \log s_{h}-\lambda \sum_{h=1}^{K}\left[1-\exp \left(-\tau_{h}\right)\right]^{\theta} \\
& -\sum_{h=1}^{K} \tau_{h}+(\theta-1) \sum_{h=1}^{K} \log \left[1-\exp \left(-\tau_{h}\right)\right]
\end{aligned}
$$

where

$$
s_{h}=\exp \left(-w_{h}^{-\beta}\right)
$$

and

$$
\tau_{h}=\left[s_{h} /\left(1-s_{h}\right)\right]^{2} .
$$

The likelihood method and its procedures are available in the literature. 


\section{Real data modeling}

This part presents in detail two real applications of the PBX-Fr version using real observations. We will compare the obtained fit of the PBX-Fr version with the exponentiated. Fréchet. (E-Fr), Kumaraswamy. Fr (Kum-Fr) (by Mead. and Abd-Eltawab. (2014)), transmuted. Fr (TFr) (by Mahmoud. and Mandouh. 2013), beta Fr (B-Fr) the WFr (by Afify et al., (2016)), Gamma. Extended. Fr (GE-Fr) (bySilva et. al. (2013)), Marshall-Olkin. Fr (MO-Fr) (byKrishna. et al., (2013)) and Fr distributions.

The 1-st data set (Nichols \& Padgett data) consists of hundred observations of carbon fibers breaking stress given (see Nichols \& Padgett (2006)). The 2-nd data set (see Smith \& Naiylor (1987)) consists of 63 observations of the glass fibers strengths.

Consider the following criteria:

The $-2 \ell_{(\lambda, \theta, \beta)}$; AIC (Akaike Information Criterion); BIC (BayesianIC); CAIC (ConsistentIC); HQIC (Hannan-QuinnIC) and the total time test (TTT).

The TTT for two real data sets are presented below in Figure 5. From Figure 5, it concluded that the empirical HRFs of the two data is increasing (for more details see (see Aarset (1987)).). In Tables 2 and 4, we compared the PBX-Fr model with other Fr models. The PBX-Fr model gives the lowest (best) values for the AIC, BIC, HQIC and CAIC among all fitted Fr models for two data sets. Figures 6-10 display the plots of estimated PDF, estimated CDF, estimated HRF, P-P plot and Kaplan-Meier survival plot of the PBX-Fr model for the two data.

Table 2: Statistics for "breaking stress data".

\begin{tabular}{|c|c|c|c|c|c|}
\hline Model & \multicolumn{5}{|c|}{ Goodness of fit (G-O-F) criteria } \\
\hline & BIC & AIC & CAIC & HQIC & $-2 \ell$ \\
\hline PBX-Fr & $\mathbf{1 2 7 . 7 7}$ & $\mathbf{1 1 9 . 9 4}$ & $\mathbf{2 9 0 . 1 9}$ & $\mathbf{1 2 3 . 1 1}$ & $\mathbf{1 1 3 . 8 2}$ \\
\hline W-Fr & 304.93 & 294.58 & 294.94 & 298.67 & 286.52 \\
\hline E-Fr & 303.55 & 295.66 & 296.01 & 298.88 & 289.74 \\
\hline Kum-Fr & 307.56 & 297.10 & 297.52 & 301.34 & 289.13 \\
\hline B-Fr & 321.60 & 311.10 & 311.58 & 315.42 & 303.19 \\
\hline GE-Fr & 332.43 & 312.00 & 312.42 & 316.20 & 304.00 \\
\hline Fr & 353.52 & 348.31 & 348.43 & 350.38 & 344.36 \\
\hline T-Fr & 358.32 & 350.46 & 350.72 & 353.62 & 344.58 \\
\hline MO-Fr & 359.10 & 351.29 & 351.64 & 354.54 & 345.34 \\
\hline
\end{tabular}


Table 3: MLEs and SEs for "breaking stress data".

\begin{tabular}{|c|c|c|c|c|}
\hline \multirow{2}{*}{$\begin{array}{c}\text { Model } \\
\operatorname{PBX}-\operatorname{Fr}(\lambda, \theta, \beta)\end{array}$} & \multicolumn{4}{|c|}{ Estimates } \\
\hline & 4.8876 & 3.708 & 0.7184 & \\
\hline & (1.1197) & $(0.307)$ & $(0.0554)$ & \\
\hline $\mathrm{W}-\mathrm{Fr}(\alpha, \beta, \mathrm{a}, \mathrm{b})$ & 2.2231 & 0.355 & 6.9721 & 4.9179 \\
\hline & (11.409) & $(0.411)$ & $(113.811)$ & $(3.756)$ \\
\hline $\operatorname{Kum}-\operatorname{Fr}(\alpha, \beta, \mathrm{a}, \mathrm{b})$ & 2.0556 & 0.4654 & 6.2815 & 224.18 \\
\hline & $(0.071)$ & $(0.00701)$ & $(0.063)$ & $(0.164)$ \\
\hline $\mathrm{B}-\mathrm{Fr}(\alpha, \beta, \mathrm{a}, \mathrm{b})$ & 1.6097 & 0.4046 & 22.0143 & 29.7617 \\
\hline & $(2.498)$ & $(0.108)$ & $(21.432)$ & $(17.479)$ \\
\hline $\operatorname{GE}-\operatorname{Fr}(\alpha, \beta, \mathrm{a}, \mathrm{b})$ & 1.3692 & 0.4776 & 27.6452 & 17.4581 \\
\hline & $(2.017)$ & $(0.133)$ & $(14.136)$ & $(14.818)$ \\
\hline E-Fr( $(\alpha, \beta, a)$ & 69.1489 & 0.5019 & 145.3275 & \\
\hline & $(57.349)$ & $(0.08)$ & $(122.924)$ & \\
\hline T-Fr $(\alpha, \beta, a)$ & 1.9315 & 1.7435 & 0.0819 & \\
\hline & $(0.097)$ & $(0.076)$ & $(0.198)$ & \\
\hline MO-Fr( $\alpha, \beta, a)$ & 2.3066 & 1.5796 & 0.5988 & \\
\hline & $(0.498)$ & $(0.16)$ & $(0.3091)$ & \\
\hline $\operatorname{Fr}(\alpha, \beta)$ & 1.8705 & 1.7766 & & \\
\hline & $(0.112)$ & $(0.113)$ & & \\
\hline
\end{tabular}

Table 4: Statistics for "strengths data".

\begin{tabular}{|c|c|c|c|c|c|}
\hline Model & \multicolumn{5}{|c|}{ G-O-F criteria } \\
\hline & BIC & AIC & CAIC & HQIC & $-2 \ell$ \\
\hline PBX-Fr & $\mathbf{5 9 . 1 2}$ & $\mathbf{5 2 . 6 9}$ & $\mathbf{5 3 . 0 9}$ & $\mathbf{5 5 . 2 2}$ & $\mathbf{4 6 . 6 0}$ \\
\hline B-Fr & 77.2 & 68.62 & 69.34 & 72.03 & 60.65 \\
\hline GE-Fr & 78.1 & 69.61 & 70.32 & 72.90 & 61.63 \\
\hline Fr & 102 & 97.72 & 97.92 & 99.44 & 93.79 \\
\hline T-Fr & 106.5 & 100.14 & 100.51 & 102.63 & 94.19 \\
\hline MO-Fr & 108.2 & 101.74 & 102.16 & 104.26 & 95.75 \\
\hline
\end{tabular}


Table 5: MLEs and SEs for strengths data.

\begin{tabular}{|c|c|c|c|c|}
\hline Model & \multicolumn{4}{|c|}{ Estimates } \\
\hline PBX-Fr( $\lambda, \theta, \beta)$ & $\mathbf{4 . 8 5}$ & $\mathbf{4 . 1 9 7 1}$ & $\mathbf{0 . 8 2 8}$ & \\
\hline & $\mathbf{( 1 . 3 1 2 )}$ & $\mathbf{( 0 . 4 3 7 4 )}$ & $\mathbf{( 0 . 0 7 2 )}$ & \\
\hline & & & & \\
\hline B-Fr( $\alpha, \beta, \mathrm{a}, \mathrm{b})$ & 2.0518 & 0.6466 & 15.0756 & 36.9397 \\
\hline & $(0.986)$ & $(0.163)$ & $(12.057)$ & $(22.649)$ \\
\hline & & & & \\
\hline GE-Fr( $\alpha, \beta, \mathrm{a}, \mathrm{b})$ & 1.6625 & 0.7421 & 32.112 & 13.2688 \\
\hline & $(0.952)$ & $(0.197)$ & $(17.397)$ & $(9.967)$ \\
\hline & & & & \\
\hline $\mathrm{T}-\operatorname{Fr}(\alpha, \beta, \mathrm{a})$ & 1.3068 & 2.7898 & 0.1298 & \\
\hline & $(0.034)$ & $(0.165)$ & $(0.208)$ & \\
\hline & & & & \\
\hline $\operatorname{MO}-\operatorname{Fr}(\alpha, \beta, \mathrm{a})$ & 1.5441 & 2.3876 & 0.4816 & \\
\hline & $(0.226)$ & $(0.253)$ & $(0.252)$ & \\
\hline & & & & \\
\hline $\operatorname{Fr}(\alpha, \beta)$ & 1.264 & 2.888 & & \\
\hline & $(0.059)$ & $(0.234)$ & & \\
\hline
\end{tabular}

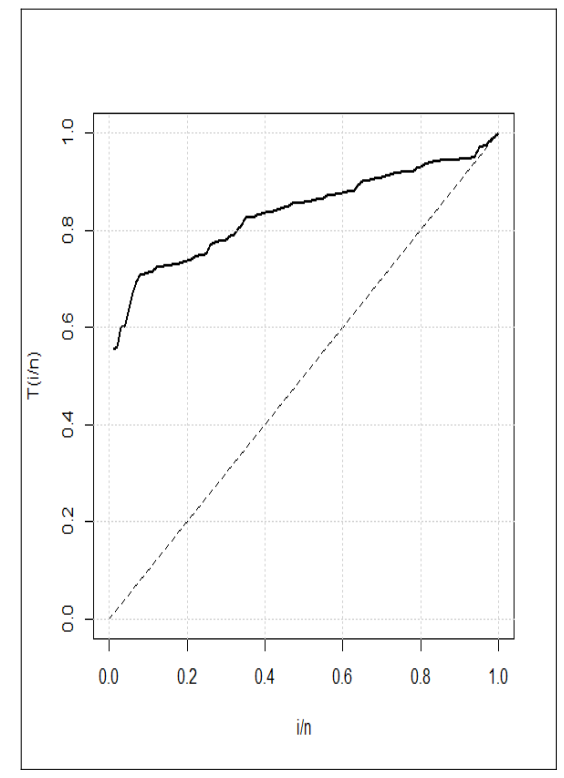

Carbon Fbers Data

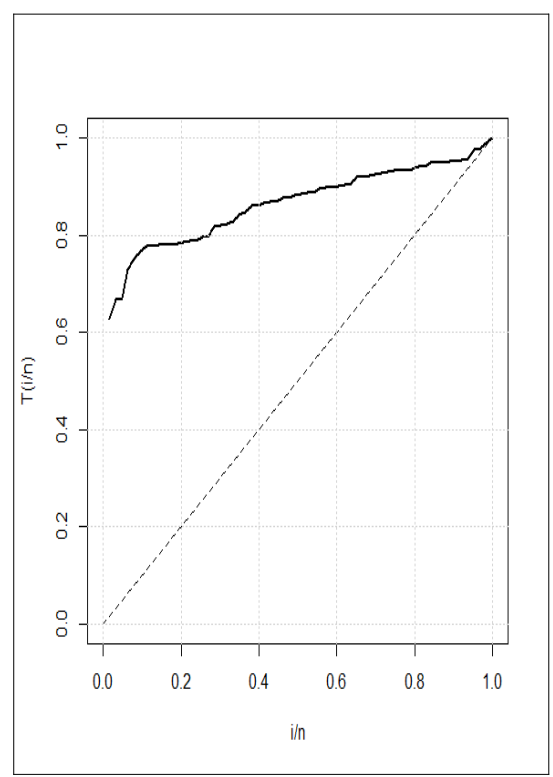

Strengths Data

Figure 5: gives the TTT plots. 


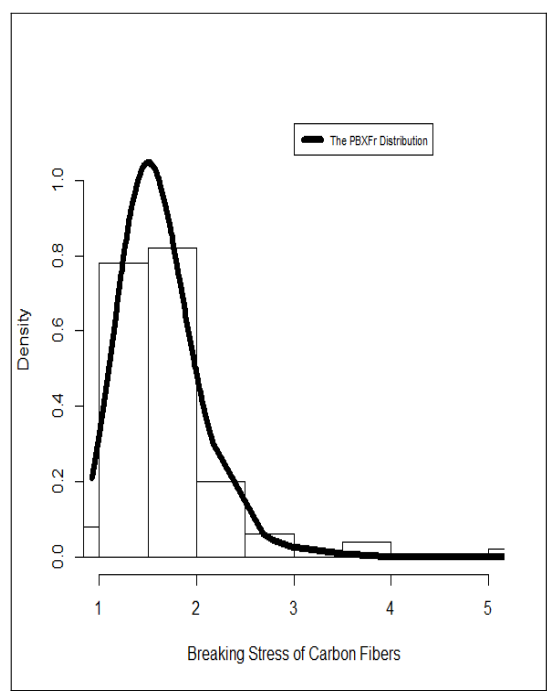

Carbon Fbers Data

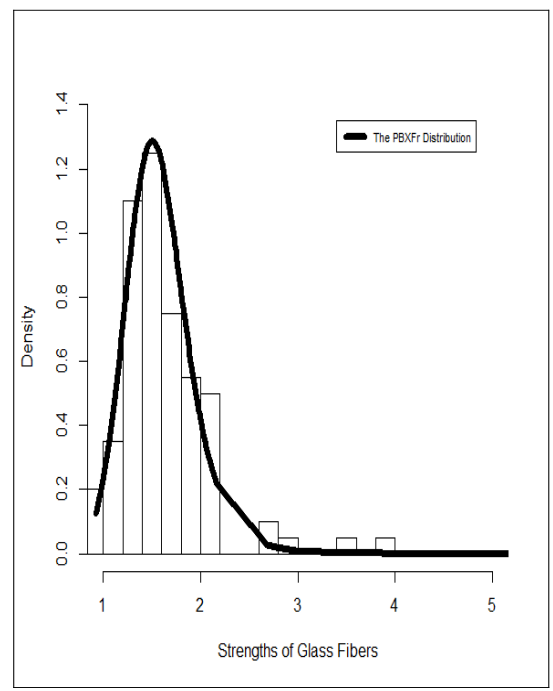

Strengths Data

Figure 6: Estimated PDFs.

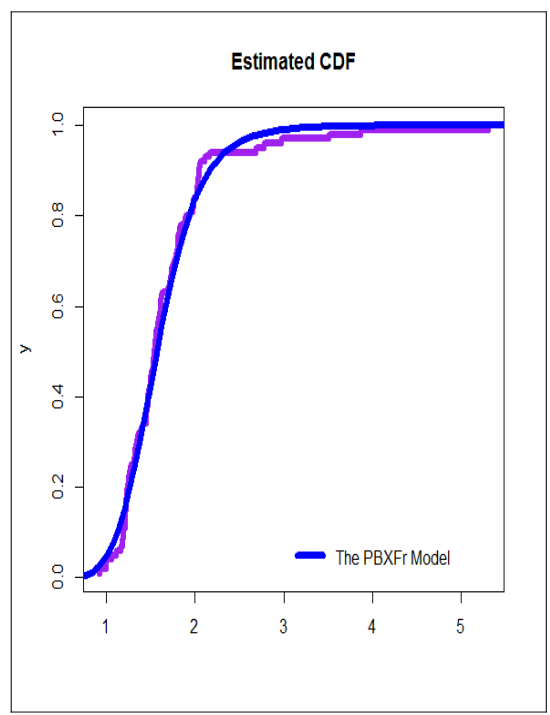

Carbon Fbers Data

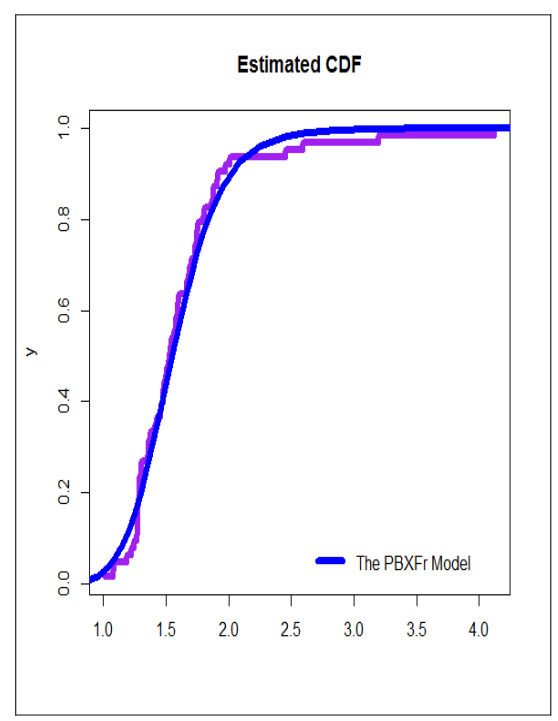

Strengths Data

Figure 7: Estimated CDFs. 


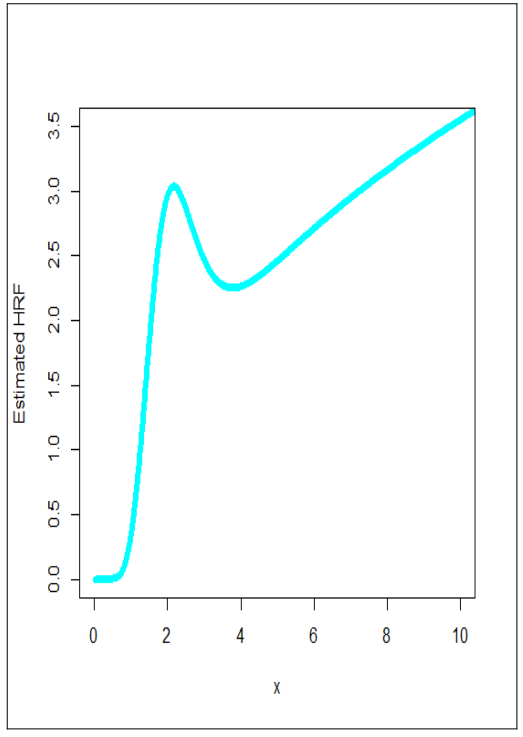

Carbon Fbers Data

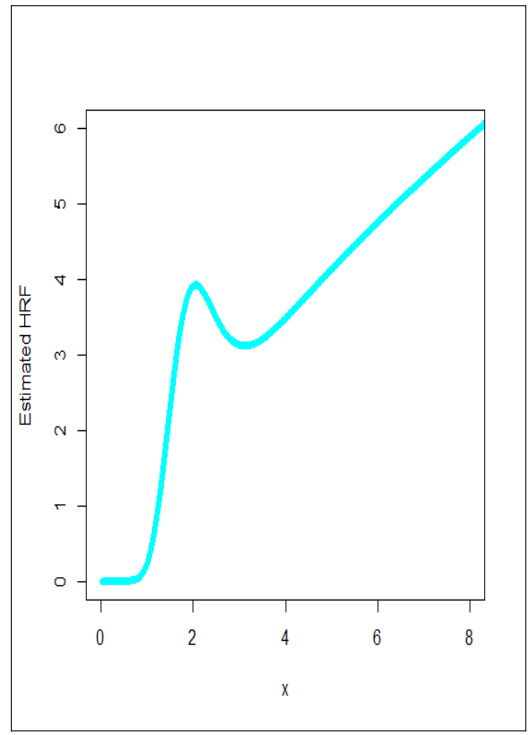

Strengths Data

Figure 8: Estimated HRFs.

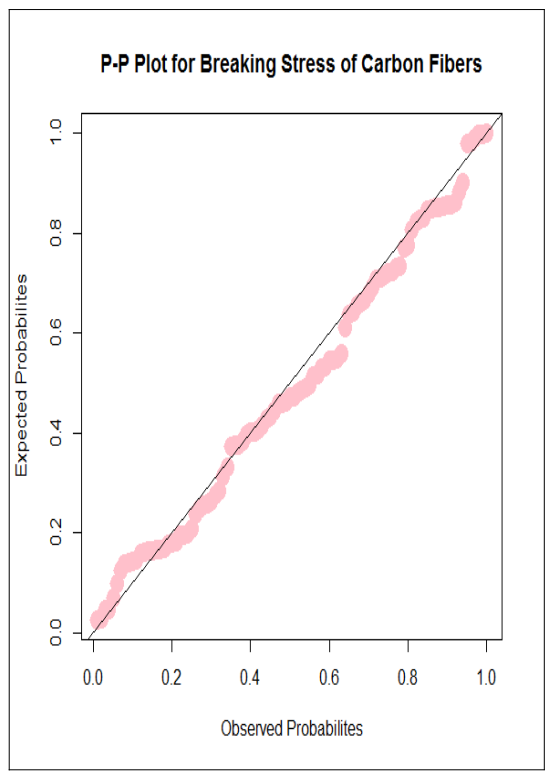

Carbon Fbers Data

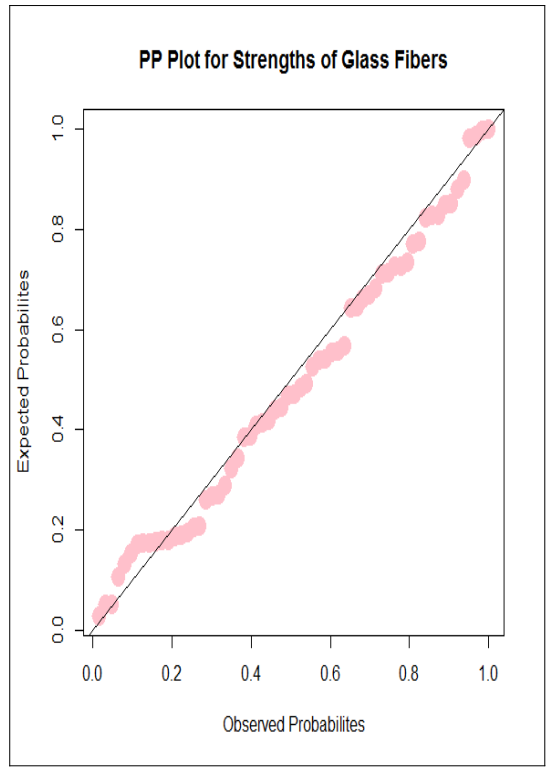

Strengths Data

Figure 9: P-P plots.

\section{Conclusions}

In this work, a new compound version of the Fréchet model is introduced and studied in detail. Some properties related to the new version are derived as well. The method of maximum likelihood method is used to estimate the unknown parameter via two real data 
applications. The new model is much better than other important competitive Fréchet models in modeling two real data sets.

\section{Acknowledgement}

This research was funded by the Deanship of Scientific Research at Princess Nourah bint Abdulrahman University through the Fast-track Research Funding Program.

\section{References}

1. Aarset, M. V. (1987). How to identify a bathtub hazard rate. IEEE Transactions on Reliability, 36(1), 106-108.

2. Afify, A. Z., Yousof, H. M. Cordeiro, G.M. \& Ahmad, M. (2016a). The Kumaraswamy Marshall-Olkin Fréchet distribution: Properties and Applications, Journal of ISOSS, 2(1), 41-58.

3. Afify, A. Z., Yousof, H. M., Cordeiro, G. M., Ortega, E. M. M. \& Nofal, Z. M. (2016b). The Weibull Fréchet distribution and its applications. Journal of Applied Statistics, 43(14), 2608-2626.

4. Brito, E., Cordeiro, G. M., Yousof, H. M., Alizadeh, M. \& Silva, G. O. (2017). Topp-Leone Odd Log-Logistic Family of Distributions, Journal of Statistical Computation and Simulation, 87(15), 3040-3058.

5. Chakraborty, S., Handique, L., Altun, E. \& Yousof, H. M. (2018). A new statistical model for extreme values: mathematical properties and applications. International Journal of Open Problems in Computer Science and Mathematics, 12(1), 1-18.

6. Cordeiro, G. M., Yousof, H. M., Ramires, T. G. \& Ortega, E. M. M. (2018). The Burr XII system of densities: properties, regression model and applications. Journal of Statistical Computation and Simulation, 88(3), 432-456.

7. Hamedani G. G., Altun, E, Korkmaz, M. C., Yousof, H. M. \& Butt, N. S. (2018). A new extended $\mathrm{G}$ family of continuous distributions with mathematical properties, characterizations and regression modeling. Pak. J. Stat. Oper. Res., 14 (3), 737-758.

8. Hamedani G. G. Rasekhi, M., Najibi, S. M., Yousof, H. M. \& Alizadeh, M., (2019). Type II general exponential class of distributions. Pak. J. Stat. Oper. Res., forthcoming.

9. Hamedani G. G. Yousof, H. M., Rasekhi, M., Alizadeh, M. \& Najibi, S. M. (2017). Type I general exponential class of distributions. Pak. J. Stat. Oper. Res., XIV(1), 39-55.

10. Korkmaz, M. C., Alizadeh, M., Yousof, H. M. \& Butt, N. S. (2018). The generalized odd Weibull generated family of distributions: statistical properties and applications. Pak. J. Stat. Oper. Res., 14 (3), 541-556.

11. Korkmaz, M. C., Altun, E., Yousof, H. M. \& Hamedani G. G. (2019). The Odd Power Lindley Generator of Probability Distributions: Properties, Characterizations and Regression Modeling, International Journal of Statistics and Probability, 8(2). 70-89.

12. Korkmaz, M. C. Yousof, H. M. \& Ali, M. M. (2017). Some Theoretical and Computational Aspects of the Odd Lindley Fréchet Distribution, Journal of Statisticians: Statistics and Actuarial Sciences, 2, 129-140

13. Mead, M. E. \& Abd-Eltawab A. R. (2014). A note on Kumaraswamy-Fréchet Distribution. Aust. J. Basic and Appl. Sci., 8, 294-300. 
14. Mubarak, M. (2011). Parameter estimation based on the Fréchet Progressive Type II censored data with binomial removals. Journal of Quality, Statistics and Reliability 2012 (2011).

15. Nadarajah, S. \& Gupta, A. K. (2004). The Beta Fréchet Distribution. Far East Journal of Theoretical Statistics, 14, 15-24.

16. Kotz, S. \& Nadarajah, S. (2000). Extreme Value Distributions: Theory and Applications. Imperial College Press, London.

17. Nadarajah, S. \& Kotz, S. (2003). The exponentiated Fréchet distribution. Interstat Electronic Journal, 1-7.

18. Nichols, M. D. \& Padgett, W. J. (2006). A Bootstrap control chart for Weibull percentiles. Quality and Reliability Engineering International, 22, 141--151.

19. Silva, R. V. D., de Andrade, T. A., Maciel, D., Campos, R. P. \& Cordeiro, G. M. (2013). A new lifetime model: The gamma extended Fréchet distribution. Journal of Statistical Theory and Applications, 12, 39--54.

20. Smith, R. L. and Naylor, J. C. (1987). A comparison of maximum likelihood and Bayesian estimators for the three-parameter Weibull distribution. Applied Statistics, 36, 358-369.

21. Yousof, H. M., Afify, A. Z., Alizadeh, M., Butt, N. S., Hamedani, G. G. \& Ali, M. M. (2015). The transmuted exponentiated generalized-G family of distributions. Pak. J. Stat. Oper. Res., 11, 441-464.

22. Yousof, H. M., Afify, A. Z., Ebraheim, A. N., Hamedani, G. G. and Butt, N. S. (2016). On six-parameter Fréchet distribution: properties and applications, Pak. J. Stat. Oper. Res., 12, 281-299.

23. Yousof, H. M., Alizadeh, M., Jahanshahi and, S. M. A., Ramires, T. G., Ghosh, I. \& Hamedani G. G. (2017). The transmuted Topp-Leone $\mathrm{G}$ family of distributions: theory, characterizations and applications, Journal of Data Science. 15, 723-740.

24. Yousof, H. M., Altun, E. \& Hamedani, G. G. (2018 a). A new extension of Frechet distribution with regression models, residual analysis and characterizations. Journal of Data Science, 16(4), 743-770.

25. Yousof, H. M., Jahanshahi, S. M., Ramires, T. G Aryal, G. R. \& Hamedani G. G. $(2018$ b). A new distribution for extreme values: regression model, characterizations and applications. Journal of Data Science, 16(4), 677-706. 